\title{
Selected Abstracts from the 6th Asia Pacific Association of Medical Toxicology, Part 1
}

\begin{abstract}
Editor's note: The following abstracts were presented at the 6th Asia Pacific Association of Medical Toxicology in Bangkok, Thailand, December 12 to 14, 2007. The range of presentations extended from sound basic science to interesting case series of unusual poisonings rarely seen outside the region. Due to space restrictions, only selected abstracts have been published here. This issue will highlight the unique abundance of pesticide and environmental poisonings including envenomations from the region. Part 2 will be published in issue 4.4 .
\end{abstract}

\section{PESTICIDE POISONINGS}

\section{PP 088. TOXICOKINETIC ANALYSES IN ACUTE CHLORPYRIFOS POISONING}

\author{
DM Roberts (1,2), M Eddleston (1,3), P Eyer (4), L Senarathna (1,5), M Fahim (1,5), NA Buckley $(1,2,5)$. \\ (1) South Asian Clinical Toxicology Research Collaboration; (2) Australian National University, Canberra, Australia; (3) Scottish Poisons \\ Information Bureau, Edinburgh, UK; (4) Ludwig Maximilians University, Munich, Germany; (5) University of Peradeniya, Peradeniya, Sri Lanka
}

Objective: To describe the concentration-time profile of chlorpyrifos in patients with acute poisoning using various methods.

Methods: Serial blood samples were provided by 59 patients with acute chlorpyrifos poisoning identified prospectively in clinical trials in Sri Lanka. Non-linear regression was applied using four relatively simple methods: (1) median value in individuals (the elimination kinetics were determined to follow a biphasic decay), (2) pooled unadjusted population data, (3) pooled population data adjusted for exposure by dividing by the AUC, and (4) global fitting of individual data using shared rate constants. The median value determined in (1) was used to inform the subsequent analyses with pooled data. A sensitivity analysis was conducted to determine the influence of the initial values on the best-fit results. The effect of relative weighting with the $1 / \mathrm{Y}^{2}$ method was also explored due to the potential influence of heteroscedasticity. The results of these analyses were compared visually and by the sum-of-squares method.

Results: The median age was 32 years and the median BChE and AChE activities on admission were $10 \mathrm{U} / \mathrm{L}$ and $53 \mathrm{mU} / \mathrm{\mu mol} \mathrm{Hb}$, consistent with severe poisoning; six patients died. The fit determined by (2) appeared to be implausible and did not describe the data so was not considered further; the results for the others are shown (Figure). Relative weighting did influence the best-fit values, in particular the terminal elimination phase, which may reflect the smaller number of samples. The sensitivity analysis suggested that the regression was not overly influenced by the initial values. The predicted disposition half-life of chlorpyrifos ranged from 1.7 to 4.2 hours and the apparent elimination half-life was 56 to 74 hours (Figure). Using the sum-of-squares measurements, the best-fit lines determined by methods (3) and (4) appeared to better describe the data. But because the half-lives predicted by each method were similar, the fits preferred by the sum-of-squares measurement may not be more informative. The ability to apply any of these methods depends on the number of patients and frequency of blood samples in each patient. The method in (3) requires further validation and appropriate samples for the AUC to be determined, while global fitting requires necessary computer software.

Conclusion: A number of relatively simple kinetic methods may be used for quantifying the concentration-time profile of a poison. In the case of chlorpyrifos these methods produced similar results, confirming that the elimination phase is prolonged.

\section{PP 006. ALUMINIUM PHOSPHIDE POISONING IN BANGLADESH: INHALATIONAL AND INTENTIONAL}

\author{
A Ghose (1), H Kabir (2), AA Sayeed (3), H Ahmed (4), A Basher (4), M Rashid (5), MR Amin (6), AM Faiz (4). \\ (1) Chittagong General Hospital, Chittagong, Bangladesh; (2) Directorate General of Health Service, Bangladesh; (3) Chittagong Medical \\ College Hospital, Chittagong, Bangladesh; (4) Dhaka Medical College Hospital, Dhaka, Bangladesh; (5) National Institute of Disease of Chest \\ Hospital, Dhaka, Bangladesh; (6) Hathazari Health Complex, Chittagong, Bangladesh
}

Objective: Aluminium phosphide (AP), a solid fumigant that produces phosphine gas, is widely used during grain preservation. The commonest mode of poisoning is ingestion due to easy availability in rural areas, whereas inhalational phosphine poisoning is 
relatively less reported. In this communication we report the first ever documented incidents of AP poisoning by oral ingestion and inhalation in Bangladesh.

Case series: Seven workers developed unusual symptoms following an overnight sleep in a food warehouse where aluminium phosphide was used as pesticide and rodenticide. No protective gear was used. Important features include dizziness, nausea, vomiting, fatigue, hypotension, atrial fibrillation, nonspecific ST and T wave changes and hypokalaemia. One patient with atrial fibrillation and hypotension was given magnesium sulphate (IV 2gm stat) and dopamine. The AF reverted within one hour and BP subsequently stabilized. All other patients recovered with supportive treatment. Follow up revealed bradycardia in two and low systolic blood pressure in four of the victims. Another adult male patient presented with respiratory distress, oliguria and jaundice three days after ingestion of a single pellet with an intention of deliberate self harm. Laboratory investigations revealed hypernatremia, acidosis, haemolysis and thrombocytopenia. Brought specimen confirmed the offending agent. He developed multi-organ failure and died 8 days later.

Conclusion: Intoxication by AP is being increasingly reported in literature as occupational or nonoccupational incidents, some with fatal outcome. Aluminium phosphide poisoning should be kept in mind in patients presenting in an unusual setting. Strict adherence to occupational safety practices is necessary to avoid such poisonings.

\title{
PP 007. UNUSUAL COMPLICATIONS OF ALUMINIUM PHOSPHIDE POISONING
}

\author{
N Sharma, S Mahi, S Varma. \\ The Department of Internal Medicine, Postgraduate Institute of Medical Education and Research, Chandigarh, India
}

Objective: To elucidate unusual complications of aluminium phosphide poisoning.

Case Series: Six patients presented with aluminium phosphide ingestion. The age range was $23-30$ years and there were 4 males and 2 females. The mean dose ingested was 3.3 gms (range $=1.5-6$ gms approximately). All cases presented with hypotension mean $\mathrm{SBP}=70 \mathrm{mmHg}(\mathrm{SBP}$ range $=$ unrecordable $-80 \mathrm{mmHg})$ and tachycardia (range $=110-140 / \mathrm{mt})$. On investigations, 1 patient had leucocytosis, all had acidosis mean $\mathrm{pH}=7.2$ (range $=7.13-7.30)$, the mean serum bicarbonate was $11.2 \mathrm{mEq} / \mathrm{L}(\mathrm{range}=3-15 \mathrm{mEq} / \mathrm{L})$ and one case had renal failure (blood urea $=136 \mathrm{mg} / \mathrm{dL}$, serum creatinine $=8.2 \mathrm{mg} / \mathrm{dL}$ ) with acute hepatitis $($ serum bilirubin $=4.8 \mathrm{mg} / \mathrm{dL}$ and $\mathrm{AST} / \mathrm{ALT}=1150 / 1310 \mathrm{U} / \mathrm{L}$ ). Appropriate fluid therapy and inotropic support (for a mean period of 2.4 days) corrected the hypotension in all. On day 3, three patients developed adult respiratory distress syndrome with bilateral transudative pleural effusion and ascites whereas two cases developed this complication on day 5 ( 1 case developed generalized body swelling and pericardial effusion). The remaining case developed massive bilateral transudative pleural effusion (that required repeated paracentesis), acute hepatitis, acute renal failure, ARDS and disseminated intravascular coagulation. An echocardiography was done in all cases and showed LV systolic dysfunction with ejection fraction ranging from 40-50\%. Electrocardiographic abnormalities that were noted were sinus tachycardia in all, ST depression in one, atrial fibrillation in one and one case had accelerated idioventricular rhythm and nonsustained ventricular tachycardia. There were 2 deaths, of case 3 and 6 . In all other patients, pleural effusion and ascites resolved within 3 days with salt and water restriction and diuretic therapy.

Conclusion: Aluminium phosphide poisoning can develop a delayed complication of pleural effusion and ascites with or without pericardial effusion and ARDS. The secondary form of capillary leak syndrome caused by phosphene is an attractive hypothesis to explain this complication.

\section{PP 008. EFFECT OF ALUMINUM PHOSPHIDE ON BLOOD GLUCOSE LEVEL}

\section{O Mehrpour (1,2), S Shadnia (1), N Chaleki (1).}

(1) Loghman-Hakim Hospital Poison Center, Faculty of Medicine, and Toxicological Research Center (TRC), Shaheed Beheshti University of Medical Sciences, Tehran, Iran; (2) Forensic Medicine Department, Faculty of Medicine, Tehran University of Medical Sciences, Tehran, Iran

Objective: Aluminum phosphide (ALP) is a solid fumigant pesticide widely used in Iran as a grain preservative. It produces phosphine gas which is a mitochondrial poison that interferes with enzymes and protein synthesis. Over the last 3 years there has been a dramatic increase in the number of cases of ALP poisoning in Iran. Although ALP can cause an increase or decrease in blood glucose levels, we felt bound to examine the role of blood glucose level as a mortality prognostic factor.

Methods: Forty-five patients with ALP poisoning due to ingestion were studied in a Loghman-Hakim Hospital Poison Center over a period of 14 months. All of them were treated with the same protocol (sodium bicarbonate, magnesium sulphate, calcium gluconate, and adequate hydration). Patients were divided to dead or alive groups and statistical comparisons were made in various parameters including blood glucose.

Results: Between March 2006 and May 2007, 45 patients with ALP poisoning were admitted; of these 47\% were female and $53 \%$ were male. The mean age of cases was $27.29 \pm 11.53$ years old with a range of $14-62$ years old. All of them were hospitalized 
in the intensive care unit. The route of exposure to ALP was deliberate ingestion in all patients. The mean of blood glucose was $222.59 \pm 20.18 \mathrm{mg} / \mathrm{dL}$ and $143.38 \pm 13.7 \mathrm{mg} / \mathrm{dL}$ in survived and expired groups respectively which was statistically significant (P value $<0.05)$.

Conclusion: Aluminum phosphide can cause either elevation, decrease or no change in blood glucose level. However in nonsurvived cases, these changes are wider and the mean of blood glucose level is higher than survived group. This hyperglycemic effect of ALP in non-survived group correlated with mortality, and suggested that the use of treatment which increases entrance of glucose to cells may be reducing oxygen consumption and may have useful role in the treatment of these patients.

\title{
PP 009. UNINTENTIONAL POISONING BY PHOSPHINE RELEASED FROM ALUMINUM PHOSPHIDE
}

\author{
S Shadnia (1), O Mehrpour (1), G Sasanian (2), M Abdollahi (1,2). \\ (1) Loghman-Hakim Hospital Poison Center, Faculty of Medicine, and Toxicological Research Center, Shaheed Beheshti University of Medical \\ Sciences, Tehran, Iran; (2) Laboratory of Toxicology, Faculty of Pharmacy, and Pharmaceutical Sciences Research Center, Tehran University of \\ Medical Sciences, Tehran, Iran
}

Background: Aluminum phosphide (ALP) is used as a grain preservative. Fumigation depends on release of phosphine gas from the ALP. Very rare reports describe unintentional exposure secondary to grain fumigation. The majority of case reports involve oral intentional ingestion.

Case report: In this presentation, we describe an accidental severe poisoning in a 35 year old woman, her 18 year old daughter and 6 year old son, caused by inhalation of phosphine gas released from 20 tablets of ALP stored in the 15 rice bags. The boy died 2 days after exposure before admission to hospital and any special treatment but the others were admitted approximately 2 days after inhalation. They had signs and symptoms of severe toxicity, and their clinical course included metabolic acidosis, electrocardiographic changes and hypotension. They were treated by intravenous administration of sodium bicarbonate, magnesium sulphate, and calcium gluconate. Conservative and supportive therapies in intensive care unit were also provided. The patients survived following treatment and supportive care and were discharged after 3 days and followed up for one week after discharge.

Discussion: ALP is absorbed rapidly by inhalation, dermal exposure or ingestion. Both pesticide applicators and individuals in the vicinity of application are at the risk of accidental exposure and injury from phosphine inhalation; however, acute accidental poisoning happens rarely. The high mortality of ALP should raise the attention of the physicians to the problem of ALP poisoning and also necessitates the awareness of the public to the hazard of this poison. The extensive use of this pesticide poses the hazard of acute accidental and intentional exposure. Among our cases one of the individuals that had no treatment died; the two others that were admitted and treated in hospital recovered. Acute inhalation of phosphine gas if it is not diagnosed and treated can be surely lethal.

\section{PP 017. ACUTE HUMAN SELF-POISONING WITH THE SUBSTITUTED AROMATIC (ORGANOCHLORINE) FUNGICIDE CHLOROTHALONIL- TETRACHLOROISOPTHALONITRIL}

\author{
S Shahmy (1), T Suhitharan (1), L Sriskandarajah (1), AH Dawson (1,2). \\ (1) South Asian Clinical Toxicology Research Collaboration, University of Peradeniya, Peradeniya; (2) School of Population and Health, University \\ of Newcastle, Australia
}

Objective: Chlorothalonil is a broad spectrum tetrachloroisopthalonitril (organochlorine) fungicide that disturbs the glycolysis and energy production by conjugation with thiols from germinating fungus cells. Chlorothalonil has been produced since the 1960 s. Regulatory tests showed it to have the potential to be a weak skin sensitiser. It is well absorbed orally and via inhalation but poorly absorbed dermally. There have been no published case reports of poisoning due to ingestion of chlorothalonil. We report the case series of chlorothalonil poisoning due to self ingestion and a single case of occupational inhalation exposure. Previous publications of human exposure are limited to 5 confirmed (patch testing) case reports of dermatitis and a single case report of anaphylaxis and two confirmed case reports (inhalational testing) of asthma due to occupational exposure.

Methodology: Clinical and biochemical data were collected prospectively from all patients with chlorothalonil poisoning as part of a multicentre observational cohort of human poisoning in Sri Lanka.

Case series: There were six patients with self ingestion and one patient with occupational inhalation of chlorothalonil. All cases were reported to a single study centre situated in the tea districts of the central hills of Sri Lanka. Most common clinical symptoms 
following self ingestion were burning sensation on mouth and throat, difficulty in swallowing, burning sensation in epigastric region and vomiting. One patient developed mild oral ulceration. One patient developed a self limited ( $<2$ minutes) single episode of generalized tonic clonic seizure. The single patient with occupational inhalation complained of difficulty in breathing with no additional sequelae. GCS was normal in all patients except for one patient who had a seizure (GCS13/15) and another with mild oral ulcer GCS (11/15). All patients remained haemodynamically normal. All patients received supportive care and the median hospital stay was 2 days. There were no deaths.

Conclusions: Deliberate self harm with chlorothalonil ingestion is rare. Human toxicity appears to be mild but larger case series are required.

\title{
PP 025. PLASMA SURFACTANT D IN PATIENTS FOLLOWING ACUTE PARAQUAT INTOXICATION
}

\author{
H-W Gil, J-O Yang, E-Y Lee, S-Y Hong. \\ Department of Internal Medicine, Soonchunhyang University Cheonan Hospital, Cheonan, Republic of Korea
}

Objective: Free radical-induced lung injury is a major problem of paraquat intoxication. Serum surfactant protein D (SP-D) reflects the severity of various lung diseases. The purpose of this study was to investigate the changes in plasma SP-D concentrations and to correlate disease severity with SP-D concentrations in patients with acute paraqaut intoxication.

Method: Twelve paraquat intoxicated patients participated in this study. Their paraquat exposure was assessed by their plasma's paraquat level. Serial plasma SP-Ds were measured by ELISA.

Results: SP-D was decreased two and three days after the initial measurement within 2 to 72 hours of ingestion. There was no difference in initial SP-D levels between survivors and non-survivors. The SP-D test revealed a significant positive correlation between the SP-D level and $\mathrm{PaO}_{2}(\mathrm{r}=0.384, \mathrm{p}=0.003, \mathrm{~N}=57)$.

Conclusion: SP-D did not predict the likelihood of survival, but it was positively correlated with $\mathrm{PaO}_{2}$. This finding suggests that low concentrations of plasma SP-D could reflect hypoxia due to free radical-induced injury.

\section{PP 027. ACUTE INTENTIONAL SELF-POISONING WITH A SELECTIVE HERBICIDE BISPYRIBAC SODIUM (BPS): A PROSPECTIVE OBSERVATIONAL STUDY}

\author{
M Ashrafdeen, M.Shukry, A Dawson, F Mohamed. \\ SACTRC Faculty of Medicine, University of Peradeniya, Sri Lanka
}

Background: BPS is a selective, systemic post-emergence herbicide.

Objective: To describe the symptoms and primary outcomes from intentional poisoning with BPS herbicide.

Method: Data was collected prospectively from 04th June 2002 to 31st July 2007 discharge or death. Plasma samples were taken on admission in all poisoned patients to be analysed in the future. Ingestion of BPS was established from the bottle and/or label brought to the hospital or by verbally communication from patient or a relative who witnessed the event.

Results: There were 73 cases with a history of BPS ingestion. Gastric lavage was carried out in 1 patient and forced emesis in 35 patients. Multiple dose of charcoal, single dose of charcoal and atropine were given respectively for 12, 9 and 3 patients. The median hospital stay, estimated volume ingested and time to present to hospital since ingestion were 3 days, $30 \mathrm{ml}$, and 3 hours respectively. The clinical features are diarrhoea, vomiting and epigastric burning (See table 1), and the cardiovascular examination findings are presented in table 2. Eleven patients recorded with epigastric pain on admission became normal on following day. Two patients had abnormal pulse on admission that became normal following day. Ten patients had a reduced consciousness on admission (GCS 6-14). These resolved within a day, and may reflect co-ingestion of alcohol. One patient had pinpoint pupils and five patients had 5 to 6 pupil sizes on admission, these resolved within few hours. No patient had cyanosis on admission and throughout the hospital stay. Eight patients were reported to have had crepitations on admission but after a few hours these resolved in most of these patients and only one patient had crepitations up to 9 days. No patient required intubation or intensive care treatment. All patients were given symptomatic treatment and supportive care. There were 2 deaths in the 73 cases of BPS poisoning, and the case fatality was $2.73 \%$ (95\% CI: 0.0018 to 0.1002 using Wald method).

Deaths: A 32 year old man admitted 13 hours after ingesting unknown amount of Bispyribac sodium with co-ingestion of alcohol. Admission signs GCS was 12/15, pulse 84/min, BP 100/70, pupil size 4 of both eyes. Died 4 hours after admission. A 43 year old man admitted 3 hours after ingesting $90 \mathrm{ml}$ of Bispyribac sodium. Forced emesis was done soon after admission. On admission, that GCS was 13/15, pulse 92/min, BP 170/90, pupil size 2 of both eyes. The patient died within 3 hours from the admission. 
Discussion: BPS poisoning has not been previously described. The main clinical features are gastrointestinal in nature; some of these symptoms may relate to the forced emesis that was carried out in over half of the cases in the series. No cause of mechanism for death was identified in the two fatal cases.

Conclusion: These data suggest that the BPS is a relatively safe overdose when compared with other herbicides such as paraquat (case fatality $>70 \%)$, glyphosate (3.4\%), MCPA (5\%) and propanil (10.5).

\title{
PP 034. REGULATION OF TOXIC CHEMICALS IN SRI LANKA AND ITS EFFECTS ON POISONING
}

WDAS De Silva.

National Poisons Information Centre, Colombo, Sri Lanka

Objective: To highlight the regulatory mechanism of chemical imports in Sri Lanka and to review its impact on morbidity and mortality of poisoning.

Method: Review of rules and regulations, international conventions/protocols on import, usage and disposal of chemicals along with literature review.

Results: Sri Lanka is predominantly an agricultural country. According to the Registrar of Pesticides, consumption of pesticides in Sri Lanka had a 1.7 fold increase in annual consumption in 2005 than in 1995. Imports of technical grade materials of pesticides had increased by 5.3 times whereas formulation imports increased by 11.4 fold in 2005 compared to 1995. The free economy policy encouraged the imports of pesticides including prohibited pesticides such as DDT and Endrin through open general license. The Control of Pesticides Act No. 33 of 1980 regulates imports and other concerns of pesticides. The Registrar of Pesticides is the sole authority implementing the laws and regulations under this act. The Pesticides Technical Advisory Committee advises Registrar of Pesticides (ROP) on policy and technical matters. The use of WHO class 1a and 1b pesticides for pest control in Sri Lanka was banned since 1995. Stockholm convention on POPs and Rotterdam convention on Prior Informed Consent Procedure was implemented through ROP. Vienna convention and Montreal protocol on Ozone Depleting Substances as well as the Basel Convention for Chemical Safety and Trans-Boundary Movement of Hazardous Wastes and their disposal are implemented through the Central Environmental Authority (CEA). The import and use of all the above chemicals will be monitored and regulated through the CEA. Imports and Exports Control Act regulates imports of chemicals to Sri Lanka. HS coding system enables the custom to levy duties/ tariffs as well as screening banned, restricted or registered for use pesticides and other chemicals. Custom ordinance has a monitoring effect on all imports. These rules and regulations have a positive impact on reduction of suicides in Sri Lanka indicated by a $50 \%$ reduction in 2005 when compared with 47 per 100,000 populations in 1995. Morbidity shows 1.5 fold increase while mortality shows 1.3 fold reduction in 2005 compared to 1995. Toxic effects of pesticides was the 6th leading cause of hospital deaths in 1995 . In 2005 it is the 11 th cause.

Conclusion: An effective mechanism regulating the import and use of highly toxic pesticides and other chemicals will reduce the mortality of poisoning.

\section{PP 036. DETERMINATION OF DIAZINON RESIDUE IN MELON AND CUCUMBER BY SOLID-PHASE EXTRACTION AND GAS CHROMATOGRAPHY-MASS SPECTROMETRY}

\author{
M Moallem, A Moallem, M Balali-Mood, M P-H Khosro Abdi, B Sedegheh Khashayarmanesh, G Abohosine, \\ $M$ Farshid Mohseninia, M Namaei Ghasemi. \\ Medical Toxicology Centre, Imam Reza Hospital, Toxicology Department of Pharmacy School
}

Introduction: Diazinon is a moderately toxic broad-spectrum organophosphate, with a LD50 of 350 to $400 \mathrm{mg} / \mathrm{kg}$ for humans. It is largely used for pest control in Iran. As an acetyl cholinesterase inhibitor (ChE) it inhibits acetylcholine (Ach) hydrolysis at the never endings. Accumulation of Ach stimulates muscarinic and nicotinic receptors causing morbidity and mortality. Diazinon is absorbed through the skin and gastrointestinal tract and is rapidly metabolized within a few hours.

Objective: Diazinon residue in melons and cucumbers has recently been a health concern in Iran. It was thus aimed to investigate Diazinon residues in melon and cucumbers.

Method: Twenty melons and cucumbers were randomly purchased from the market in Mashhad. The extraction procedure was in HR-P columns preconditioned with methanol and water. The subsequent elution of diazinon was performed with a mixture of hexane-ethyl acetate $(1: 1, \mathrm{v} / \mathrm{v})$ prior to the determination by GC-NPD and GC-MS/MS. Standards were prepared spiking blank juice samples to contract the observed matrix effect. Accuracy $(<9.6 \%)$, precision $(<7.1 \%)$, specificity (quantitative, no peaking intraction), sensitivity (LOD $0.1 \mathrm{ng} / \mathrm{ml}$ and LOQ $5 \mathrm{ng} / \mathrm{ml}$ ) and reproducibility $(>95.4 \%)$ were acceptable. Detection limit was $1 \mathrm{ng} / \mathrm{ml}$. Linear relation with good correlation coefficients $(\mathrm{R} 2>0.99)$ was obtained. 
Results: Mean recovery for diazinon was $95.4 \%$ with relative standard deviation lower than $9 \%$ in the concentration range of $5-200 \mathrm{ng} / \mathrm{ml}$. Mean and SD of diazinon in melons was $107.64 \pm 38.5 \mathrm{ng} / \mathrm{kg}$. Diazinon was not detected in cucumber samples. It was later confirmed that diazinon was not used for the crops of these cucumbers.

Conclusions: GC-NPD and MS/MS was developed to determine residue of diazinon in melons and cucumbers. The GC-MS/MS analytical method showed a high efficacy for determination of diazinon residues in the fruits.

\title{
OP 024. BEDSIDE SCREENING TEST TO DETERMINE METHAEMOGLOBIN SEMIQUANTITATIVELY IN PATIENTS WITH PROPANIL SELF-POISONING
}

\author{
HSF Shihana (1,2), DM Dissanayake (1), A Dawson (2), University of Peradeniya, Sri Lanka. \\ (1) Department of Pathology; (2) South Asian Clinical Toxicology Research Collaboration, Faculty of Medicine
}

Propanil (3, 4-dichloropropioanilide) is a selective contact anilide herbicide used in rice, wheat and potato cultivation to control broadleaves and grass weeds. Propanil-induced methaemoglobinemia is mediated by enzymatic hydrolysis of propanil to 3, 4-dichloroaniline which is able to directly oxidize haemoglobin $(\mathrm{Hb})$ to methaemoglobin (metHb) making the haem moiety incapable of carrying oxygen. Treatment of propanil-induced methaemoglobinemia in Sri Lanka is complicated by the lack of laboratory facilities.

Objective: To discover a bedside screening test for detection of the amount of metHb in patient with propanil self poisoning at peripheral hospitals where laboratory facilities are not available.

Method: $10 \%$ to $100 \%$ metHb blood samples were prepared and the amount of metHb in the blood sample was measured using the method described by Evelyn and Malloy. Red-brown coloured blood was observed from 10\% to 100\% metHb blood samples. The prepared methaemoglobin samples were subsequently used to develop a bedside test, which could measure the level of methaemoglobin in the blood. A series of metHb samples were prepared and one to two drops were placed on piece of absorbent and scanned. The colour grade from red to brown was measured using an image program: Image $1.37 \mathrm{v}$ (e.g., an area was selected and the median RBG [red, blue, green] values were calculated after processing with median filter with 25 pixels in three times). According to the change in RBG value, a colour code was prepared.

Results: The absorbance at $630 \mathrm{~nm}$ correlates linearly with metHb levels. There is an inverse relationship between colour grade and metHb content with the correlation coefficient of 0.9938 . Variability in $\mathrm{Hb}$ levels was not associated with significant variation in the colour grade and would not significantly affect the metHb colour grade for various patients.

Conclusion: The result shows the possibility of developing a colour code for detection of the amount of methaemoglobin for as a bedside test to guide treatment.

\section{OP 038. CARDIAC INVOLVEMENT IN CHOLINESTERASE POISONING}

\author{
A Bhalla (1), S Anand (1), S Singh (1), Y Sharma (2), U Nahar (3), D Singh (4), Post Graduate Institute of Medical \\ Education and Research, Chandigarh, India. \\ (1) Department of Internal Medicine; (2) Department of Pathology; (3) Department of Cardiology; (4) Department of Forensic Medicine
}

Introduction: Electrocardiographic (ECG) changes have been noticed in those patients who die of acute anticholinesterase poisoning and vary from non-specific changes to fatal ventricular arrhythmias. Autopsy studies have confirmed acute toxic myocarditis and myocardial necrosis on histopathology.

Aims and Objectives: The present study was undertaken to evaluate various cardiac effects in patients with acute anticholinesterase poisoning including electrocardiographic and echocardiographic changes and to correlate these changes with histopathological changes of the heart at autopsy.

Patients and Methods: 44 patients with acute anticholinesterase poisoning occurring between January 1 st 2006 and June 2007 were included in the study. The diagnosis of anticholinesterase poisoning was based on history of ingestion or accidental exposure, clinical features and estimation of butyryl cholinesterase enzyme activity in plasma. Complete clinical profile of the patients was noted and ECG recorded at the time of admission. Haemodynamic parameters were monitored closely. Patients with acute organophosphorus poisoning were treated with atropine and 2-PAM (pralidoxime) whereas those with carbamate poisoning were managed with atropine only. Patients were managed with other supportive measures like mechanical ventilation, care of secretions, intravenous fluids and inotropes as needed.

Observations: 76 patients presented with acute anticholinesterase poisoning and 44 were included in the study (58\%). $66 \%$ were males and 34\% females. The mean age was 26.7 ( \pm 9.6$)$ years. In 39\% patients the compound was unknown. Carbamate was identified $18 \%$ patients. Among the organophosphates, chlorpyrifos was the most common poisoning, identified in $11 \%$ cases. In 
$82 \%$ patients the route of exposure was by ingestion. The circumstance of poisoning was with suicidal intent in $48 \%$ patients. Tachycardia was observed in $66 \%$ and $20 \%$ presented with hypotension. Baseline abnormal electrocardiogram was observed in $84 \%$ patients. Sinus tachycardia was the most common abnormality noted in $66 \%$ patients on ECG. Echocardiography could be carried out in only $84 \%$ patients and abnormalities were present in $27 \%$ patients. Mild valvular abnormalities and left ventricular diastolic dysfunction (mild) were each seen in $13.5 \%$ patients. There were 13 deaths in the study group. On autopsy, myocardial blotching and discolouration were noted in 12 patients (92.2\%). All 13 patients had interstitial oedema and vascular congestion of varying degrees. Interstitial inflammation was seen in 8 patients (61.5\%). Thrombus was noted in 6 patients (46.1\%) and pericarditis in 4 patients (30.8\%). Patchy myocarditis and focal interstitial fibrosis were each noted in 15.3\% patients. ECG abnormalities were predominantly seen in patients with interstitial oedema and vascular congestion but none of the ECG abnormalities correlated with histopathological findings.

Conclusions: Cardiac abnormalities on ECG and echocardiography are common in patients with acute anticholinesterase poisoning but do not correlate very well with histopathological changes on autopsy.

\title{
OP 039. LONG-LASTING EFFECTS OF ACUTE ORGANOPHOSPHORUS INSECTICIDE POISONING ON COGNITIVE PROCESSING OF VISUAL INFORMATION
}

\author{
T Dassanayake, V Weerasinghe, U Dangahadeniya, K Kularatne, A Dawson, N Senanayake. \\ South-Asian Clinical Toxicology Research Collaboration, Faculty of Medicine, Peradeniya, Sri Lanka
}

Objective: Acute organophosphorus (OP) poisoning may lead to long-term neuropsychological impairment. However, the manifestations reported in literature show numerous inconsistencies, highlighting the need for more objective and quantitative measures of cognitive functions. This study was conducted to determine whether acute OP insecticide poisoning leads to long-lasting impairment of objective parameters of cognitive processing visual information.

Methods: The first part of this research was a case-control study where a group of patients who had recovered from the cholinergic phase of OP poisoning $(\mathrm{n}=44)$ were compared with two age- and sex-matched control groups, viz. healthy controls $(\mathrm{n}=43)$ and patients with paracetamol overdose $(n=11)$. The second part was a prospective study where the OP poisoned patients were followed up after 6 months of poisoning and the findings were compared with their initial measurements. The tests used to assess visuomotor information processing were simple visual reaction time, recognition visual reaction time, visual evoked potentials (VEP) and motor evoked potentials. The term "cognitive processing time (CPT)" was used to denote the time taken from initial cortical perception of a stimulus to initiation of descending motor impulses. CPT of each type of visual reactions was calculated by subtracting the sum of the visual impulse duration and the motor impulse duration from visual reaction time $(\mathrm{CPT}=$ visual reaction time $-[\mathrm{P} 100$ VEP latency + total motor conduction time]) (Figure 1).

Results: OP poisoned patients showed significant delays in CPT of simple visual reactions $\left(\mathrm{CPT}_{\mathrm{SVR}}\right)$ and $\mathrm{CPT}$ of recognition visual reactions $\left(\mathrm{CPT}_{\mathrm{RVR}}\right)$ compared to both control groups. Comparison of the initial and follow-up findings of the patients revealed a sustained impairment in $\mathrm{CPT}_{\mathrm{SVR}}$ and an improvement in $\mathrm{CPT}_{\mathrm{RVR}}$. Visual and motor conduction latencies were similar between the groups and between the two assessments of the OP intoxicated patients (Table 1).

Conclusions: OP insecticide poisoning appears to slow the cognitive processing of visual information. These effects persist beyond cholinergic phase of poisoning, and the deficits in cognitive processing in simple visual reactions appear to be persistent even 6 months after poisoning. Being an objective measure of cognitive processing, CPT could also be used to assess neurocognitive deficits that follow other intoxications or disease states.

\section{OP 040. INFLUENCE OF GENETIC VARIATION ON PON1 ACTIVITY AND SUSCEPTIBILITY TO ORGANOPHOSPHATE TOXICITY}

\author{
S Jintana (1), K Sming (1), Y Krongtong (2), S Thanyachai (1), Mahidol University, Bangkok, Thailand. \\ (1) Department of Medicine, Faculty of Medicine, Ramathibodi Hospital; (2) Department of Pharmacology, Faculty of Science
}

Objective: The hydrolysis of toxic oxon metabolites of organophosphates (OPs) by paraoxonase (PON1) is one of the important factors determining their toxicity to humans. Thus, this study was to determine genetic variation of PON1, PON1 activity and effect on cholinesterase activity.

Methods: Cholinesterase and PON1 activities were measured once for the control group $(\mathrm{n}=30)$ and twice for exposed $(\mathrm{n}=90)$ groups, over high and low exposure periods. Three polymorphisms of PON1 (T-108C, Q192R and L55M) were identified, by PCR-RFLP method, only in the exposed group. 
Results: The results demonstrated that AChE activity in both high $(20.73 \mathrm{U} / \mathrm{gHb})$ and low exposure periods (29.8 U/gHb) of the exposed group were significantly different from control group (38.98 U/gHb, p $<0.01)$. For BuChE activity, the exposed group also showed the statistically lower level in both periods (high exposure period; $3.73 \mathrm{U} / \mathrm{mL}$ and low exposure period; $4.91 \mathrm{U} / \mathrm{mL}$ ) than those in the control group $(5.96 \mathrm{U} / \mathrm{mL})$. The PON1 genotype and allele frequencies of PON1 were similar to those found in the Asian population and did not deviate from Hardy-Weinberg equilibrium expectation. The PON1 Q192R was in linkage disequilibrium with the PON1 L55M and T-108C. PON1 activities toward three substrates (paraoxon, phenylacetate and diazoxon) were partly determined by genetic variation, as seen in each genotype and haplotype individual. There was a significant relationship between observed high paraoxonase activity haplotype (211) with high BuChE activity.

Conclusion: A functional defect of PON1 or reduction in PON1 level is expected to affect cholinesterase particularly susceptible to anti-cholinesterase exposure. Individuals with low PON1 activity in that genotype are more susceptible to OP toxicity than those with high activity PON1.

\title{
OP 041. BIOCHEMICAL CORRELATIONS OF ACUTE CHLORPYRIFOS POISONING
}

\author{
P Jayawardane, A Dawson, N Senanayake, V Weerasinghe, N Buckley, P Eyer. \\ South Asian Clinical Toxicology Research Collaboration, Faculty of Medicine, University of Peradeniya, Sri Lanka
}

Introduction: We previously observed that intermediate syndrome (IMS) is a spectrum disorder with diagnostic features on repetitive nerve stimulation (RNS).

Objective: The objective was to determine whether there is a correlation between IMS and admission red blood cell acetylcholinesterase level (RBC-AChE), butyrylcholinesterase level (BChE) and serum organophosphate (OP) level in patients with acute chlorpyrifos poisoning.

Method: A prospective case series of 59 consenting symptomatic patients with acute chlorpyrifos poisoning were assessed. Repeated neurological examinations and RNS studies were done. RBC-AChE, BChE and OP levels were assessed on admission in 36, 59 and 59 patients respectively. The Mann Whitney U test was used to compare the differences.

Results: Thirty of 59 patients developed electrophysiological abnormalities associated with muscle weakness indicating intermediate spectrum disorder. From the 30 intermediate spectrum patients (Group I) admission RBC-AChE, BChE and serum OP levels were assessed in 19, 30 and 30 patients respectively. From the 29 non-intermediate spectrum patients (Group II) admission RBC-AChE, BChE and serum OP levels were assessed in 17, 29 and 29 respectively. There was no significant difference in RBC-AChE level between Group I and Group II ( $\mathrm{p}=0.2885)$. There was a significant difference in admission BChE level between the 2 groups $(\mathrm{p}=0.0196)$. Admission serum chlorpyrifos concentration was also significantly different between the 2 groups $(p=0.0059)$.

Conclusion: The amount of OP absorbed and the extent of BChE inhibition on admission seems to have an influence on the development of intermediate spectrum disorder. Admission RBC-AChE does not show a significant difference between the two groups. Use of pralidoxime prior to RBC-AChE measurement might reduce the association as pralidoxime does not readily reactivate BChE at the normal therapeutic concentrations but it reactivates RBC-AChE. Serial RNS studies currently remain the best way of identifying patients at high risk of IMS.

\section{OP 047. ACUTE INTENTIONAL SELF-POISONING WITH A SELECTIVE HERBICIDE FENOXAPROP-P-ETHYL (FPPE): A PROSPECTIVE OBSERVATIONAL STUDY}

\author{
M Shukry (1), M Ashrafdeen (1), C Palagasinghe (1), A Dawson (1), I Bandara (1), N Buckley (1,2), F Mohamed (1).
}

(1) SACTRC Faculty of Medicine, University of Peradeniya, Sri Lanka; (2) Department of Clinical Pharmacology and Toxicology, Canberra Clinical School, Australia

Background: FPPE is a selective aryloxy phenoxy-propionate herbicide. It inhibits fatty acid biosynthesis; by inhibiting acetyl CoA carboxylase enzyme found in plant chloroplasts and mammalian liver.

Objective: Our objective is to describe the symptoms and primary outcomes from intentional poisoning with FPPE herbicide.

Method: All FPPE poisoned patients presenting to the two General Hospitals had data prospectively collected by trained doctors until death or discharge between May 2002 to December 2006. From the majority of patients a plasma sample taken on admission to confirm exposure and were asked to consent for multiple samples. Ingestion of FPPE was established from the bottle and/or label brought to the hospital or verbally from patient or a relative who witnessed the event. The plasma samples have not yet been analyzed.

Results: There were 54 cases with a history on FPPE ingestion out of a total of 12,892 (0.4\%) poisoning patients. The median hospital stay, estimated volume ingested and time to present to hospital since ingestion were 2 days, $30 \mathrm{ml}$, and 4 hours respectively. Forced emesis was done for 37 patients and 5 patients had undergone gastric lavage. These may have contributed to high rate of vomiting and epigastric burning reported. Four patients had a reduced consciousness on admission (GCS 9-14), resolving within a 
few hours, thought to be due to co-ingestion of alcohol. All patients were given symptomatic and supportive care. There were no subsequent adverse cardio-vascular, respiratory or neurological events needed for ventilation or intensive care treatment. The case fatality was zero (95\% CI: 0 to $6 \%)$

Conclusion: There is no published literature on human cases of FPPE. These data suggest that the FPPE is a safe herbicide in acute poisoning particularly compared with other herbicides such as paraquat (case fatality > 70\%), glyphosate (3.4\%), MCPA (5\%) and propanil (10.5\%).

\title{
OP 002. ATROPINE AND GLYCOPYRROLATE IN ORGANOPHOSPHATE POISONING
}

\author{
S Senthil Kumaran, VP Chandrasekaran. \\ Division of Clinical Toxicology, Department of Accident, Emergency and Critical Care Medicine, Vinayaka Mission Kirupananda Variyar Medical \\ College Hospital, Salem, Tamilnadu
}

Background: Unintentional and intentional organophosphate (OP) poisonings continue to be a significant cause of morbidity and mortality in our country. Conventional treatment with atropine may lead to CNS toxicity, although control of secretions may still be inadequate.

Aim: To study the effectiveness of atropine with glycopyrrolate and atropine alone in organophosphate poisonings.

Methods: A prospective randomized double-blinded, placebo-controlled trial done in an emergency department of VMKV Medical College Hospital. Patients who consumed OPC were included. Pregnant women, hypothermic adult, poly pharmacy and along with alcohol were excluded. Patients with OPC poisoning, received either atropine and glycopyrrolate or atropine and matching placebo as a bolus through a peripheral IV line. All other aspects of treatment were carried out as per guidelines.

Results: 72 victims were involved during six month period 36 were belong to study group and remaining were in control group. There were no significant differences in demographic data, time of arrival and time to start treatment. After administration of the study drug. Mean duration in ventilator was 4.2 in study group and 7.4 in control group. The mean ICU stay was 6 days in control group and 11.4 days in control group. CNS toxicity occurred in $40 \%$ of control group and $4 \%$ of study group. Intermittent syndrome developed in $10 \%$ of control group and $2 \%$ of study group. Development of respiratory tract infection seen in 30\% of control group and $10 \%$ of study group.

Conclusion: Addition of glycopyrrolate appears to be a promising new intervention in the management of OPC poisoning.

\section{OP 003. CONVENTIONAL BOLUS DOSE VERSUS INCREMENTAL DOSE ATROPINISATION IN ORGANOPHOSPHORUS POISONING: A RANDOMIZED CLINICAL TRIAL}

\author{
M Joynal Abedin (1), M Gofranul Hoque (1), A Abu Sayeed (1), MA Faiz (2). \\ (1) Chittagong Medical College, Chittagong, Bangladesh; (2) Dhaka Medical College, Dhaka, Bangladesh
}

Objective: Severe organophosphorus compound (OPC) poisoning is an important clinical problem is many countries of the world. The rapid and effective stabilization and treatment of pesticide poisoned patients on their admission should reduce the number of early deaths. In this regard the efficacy and safety of incremental dose atropinisation followed by atropine infusion to conventional bolus dose atropinisation needs evaluation.

Method: A randomized clinical trial was conducted in the in-patient Department of Medicine, Chittagong Medical College, Chittagong, Bangladesh on 156 cases of OPC poisoning from June to September, 2006. The patients were divided into two groups. The patients randomized to conventional bolus dose regimen were treated by giving atropine 2 mg to 5 mg intravenously and this was repeated every 10 to 15 minutes until signs of atropinisation were clinically evident. The subsequent dose and frequency of atropine injections were individualized either by decreasing the dose or increasing the interval between the doses. The patients randomized to incremental dose atropinisation regimen received initial dose of 1.8-3.0 mg (3-5 amp) of atropine followed in 5 minutes by double dose if needed and so on doubling the previous dose after every 5 minutes interval till all or maximum parameters of atropinisation most importantly clear lung, heart rate $(>80 / \mathrm{min})$ and systolic blood pressure $(>80 \mathrm{mmHg})$ were evident. After initial atropinisation, patient was maintained on atropine infusion using 10-20\% of atropine required to load the patient in every hour. With each treatment regimen atropinisation was maintained for 24 to 48 hours. Other supportive treatments including use of pralidoxime were same in each treatment group.

Result: Out of 156 patients, one patient left the hospital on his own and was excluded from outcome analysis. Among the remaining 155 patients, the mortality in incremental dose atropinisation recipients was 8\% (6/75) compared to 22.5\% (18/80) in conventional bolus dose atropinisation recipients $(\mathrm{p}<0.05)$. Incremental dose atropinisation was also associated with earlier atropinisation $(23.90$ minutes vs 151.74 minutes; $\mathrm{p}<0.001)$ and less atropine toxicity $(12.0 \%$ vs $28.4 \%$; $<0.05)$. 
Conclusion: More clinical studies to determine the optimal dosing regimen of atropine is required and incremental dose atropinisation followed by atropine infusion should become the treatment of choice for management of OPC poisoning.

\title{
OP 004. A PROSPECTIVE STUDY ON TWO ATROPINE REGIMENS IN ACUTE ORGANOPHOSPHORUS AND CARBAMATE POISONING
}

\author{
PMS Perera (1), S Shahmy (1), I Gawarammana (1,3), AH Dawson (1,2). \\ (1) South Asian Clinical Toxicology Research Collaboration, Faculty of Medicine, University of Peradeniya, Sri Lanka; (2) School of Population \\ Health, University of Newcastle, Australia; (3)Peradeniya Teaching Hospital, Sri Lanka
}

Objective: There is wide variation and lack of evidence in current recommendations for atropine dosing schedules leading to subsequent variation in clinical practice. Therefore we sought to examine the safety and effectiveness of a titrated versus ad hoc atropine treatment regimen in a cohort of patients with acute cholinesterase inhibitor pesticide poisoning.

Method: A prospective cohort study was conducted in 3 district secondary referral hospitals in Sri Lanka using a structured data collection form that collected details of clinical symptoms and outcomes of cholinesterase inhibitor pesticide poisoning, atropine doses and signs of atropinisation. We compared two hospitals that used a titrated dosing protocol based on a structured monitoring sheet for atropine infusion with another hospital using an ad hoc regime.

Results: During the study 272 symptomatic patients with anticholinesterase poisoning requiring atropine were admitted to the three hospitals. Outcomes of death and ventilation were analyzed for all patients; 226 patients were prospectively assessed for atropine toxicity. Patients in the titrated dose cohort were more severely poisoned at baseline, having ingested pesticides with higher human toxicity, been transferred to hospital from a peripheral hospital, and had more clinical signs of anticholinesterase poisoning. They received less pralidoxime and atropine and were less likely to develop features of atropine toxicity such as delirium (1\% vs $17 \%)$, hallucinations ( $1 \%$ vs $35 \%$ ) or either ( $1 \%$ vs $35 \%$ ) and need for patient restraint (3\% vs $48 \%$ ) compared with the ad hoc dose regime. After adjusting for the pesticides ingested, there was no difference in mortality and ventilatory rates between protocols.

Conclusions: Ad hoc high dose atropine regimens are associated with more frequent atropine toxicity without any obvious improvement in patient outcome compared with doses titrated to clinical effect. Atropine doses should be titrated against response and toxicity. Further education and the use of a structured monitoring sheet may assist in more appropriate atropine use in anticholinesterase pesticide poisoning.

\section{OP 009. ACUTE ALACHLOR AND BUTACHLOR HERBICIDE POISONING}

YC Lo (1,2), CC Yang (2,3), JF Deng (2).

(1) Faculty of Medicine, School of Medicine, National Yang-Ming University, Taipei, Taiwan; (2) Division of Clinical Toxicology, Department of Medicine, Taipei Veterans General Hospital, Taipei, Taiwan; (3) Department of Environmental and Occupational Medicine, School of Medicine, National Yang-Ming University, Taipei, Taiwan

Objective: Alachlor and butachlor are commonly used herbicides. Data on related acute human poisonings, however, are scarce. We retrospectively analyzed the data of human alachlor/butachlor poisoning in Taiwan.

Methods: From October 1986 through February 2007, 63 alachlor and 70 butachlor poisoning cases were reported to the Taiwan National Poison Center. Their clinical data were reviewed and analyzed.

Results: Most cases intentionally ingested the herbicides. The toxicities of alachlor and butachlor were largely similar. Twentyeight out of 102 patients with oral exposure were asymptomatic, while the others developed vomiting, central nervous system depression, and other outcomes. Among patients involving other exposure pathways, gastrointestinal effects were the main manifestation. Three patients died after manifesting profound hypotension and/or coma following alachlor ingestion.

Conclusion: Alachlor and butachlor poisonings are usually of low toxicity. However, severe neurological and cardiovascular outcomes could rarely develop, especially following oral ingestion. Medical management of such poisonings is primarily supportive.

\section{OP 010. INFLUENCE OF GASTRIC DECONTAMINATION ON PATIENT OUTCOME AFTER PARAQUAT INGESTION}

MF Wilks (1), JA Tomenson (2), NA Buckley (3,4), A Dawson (4).

(1) Syngenta Crop Protection AG, Basel, Switzerland; (2) Causation Limited, Macclesfield, UK; (3) Australian National University Medical School, Canberra, Australia; (4) South Asian Clinical Toxicology Research Collaboration (SACTRC), Peradeniya, Sri Lanka 
Objective: To study the possible impact of administration of adsorbents and/or gastric lavage on survival in a cohort of patients with paraquat ingestion.

Methods: Cases from a survey of paraquat poisoning presenting to nine base and general/teaching hospitals across Sri Lanka over a 26 month period were analysed. A standardised questionnaire was used to collect details of the circumstances of ingestion, treatment and outcome. Analyses were performed using non-parametric methods (Kaplan-Meier and log rank trend tests) and semiparametric (Cox's proportional hazards with adjustment for potential confounding factors) methods.

Results: Data from 586 patients were included in the analysis. Most patients (84.5\%) were given Fuller's Earth or activated charcoal. Adsorbent treatment did not significantly affect survival in the model, nor was there evidence of an interaction with the formulation type. Gastric lavage was given to $61.9 \%$ of patients. Patients who had ingested $<30 \mathrm{ml}$ and did not survive died earlier if they received lavage $\left(\chi^{2}=8.7, \mathrm{p}=0.003\right)$, whereas lavage had no significant influence on time to death for those ingesting $>30 \mathrm{ml}\left(\chi^{2}=0.4, \mathrm{p}=0.51\right)$. When analysing for the influence of lavage on survival using Cox regression models, the hazard ratio (HR) for patients receiving lavage was 1.47 (95\% CI 0.96-2.25), but in the subgroup of patients who had ingested $>30 \mathrm{ml}$ and received lavage there was an additional multiplicative effect $(\mathrm{HR}=0.55 ; 95 \%$ CI $0.32-0.93)$, resulting in a small net beneficial effect of lavage in the higher ingestion group $(\mathrm{HR}=0.81)$. When including time to admission to a primary or study hospital in the analysis, there was no evidence of any effect of lavage for patients ingesting either low or high volumes if they were admitted later than an hour. In contrast, the association of lavage with poor outcome was more pronounced in patients who were admitted within one hour (HR $=2.53$; 95\% CI 1.01-6.36) and so was the interaction effect in the subgroup of patients who had ingested $>30 \mathrm{ml}$ (HR $=0.23 ; 95 \%$ CI $0.07-0.76$ ) showing an overall protective effect of lavage in the high ingestion group ( $\mathrm{HR}=0.58)$. However, this had only a small effect on the very low survival rate in this group.

Conclusion: Given the small number of patients receiving no adsorbent treatment, no firm conclusions can be drawn regarding its possible efficacy. Our data suggest that in this cohort of patients gastric lavage may have contributed to a poorer outcome for patients who ingested $<30 \mathrm{ml}$, whereas it may have had a small beneficial effect in patients who ingested higher volumes, in particular in those admitted to hospital within one hour of ingestion.

\title{
ENVIRONMENTAL POISONINGS
}

\section{PP 092. FATAL POISONING DUE TO AN ACCIDENTAL CONSUMPTION OF ADENIA DIGITATA - A CASE REPORT}

\author{
BSK Shetty (1), RG Menezes (1), M Shetty (2). \\ (1) Department of Forensic Medicine and Toxicology, Kasturba Medical College, Mangalore, South Canara, India; (2) Department of Forensic \\ Medicine and Toxicology, K.S. Hegde Medical Academy, Mangalore, South Canara, India
}

Background: Modeccin poisoning is a serious and potentially fatal event which results from ingestion of Adenia digitata, a plant belonging to the Passifloraceae family. Adenia digitata, commonly known as wild granadilla, contains the toxin modeccin which has a decreased capacity of protein synthesis and some cardiotoxic effect. We report a rare case of plant poisoning with Adenia digitata.

Case report: A 17-year-old boy died of rapid progressive multiple organ failure 96 hours after ingestion of Adenia digitata fruits. The victim had accidentally ingested the fruits of Adenia digitata, mistaking it for the edible passion fruit, Passiflora edulis. Post-mortem histopathological sections of the heart showed pericardial haemorrhages and congested vessels. The myocardium showed focal coagulative necrosis, hydropic swelling and contraction band necrosis. Lung parenchyma showed extensive alveolar damage, intraalveolar and interstitial haemorrhages, extensive areas of congestion and inflammatory infiltrate. Toxicological analysis of post-mortem visceral and blood samples revealed the presence of modeccin.

Conclusion: We recommend that all patients suspected of modeccin intoxication, due do its unpredictable outcome and unavailability of specific antidotal treatment, should be managed according to the principles of intensive care, irrespective of the actual degree of poisoning.

\section{PP 094. NEUROTOXIC MANIFESTATIONS OF SNAKE BITE IN BANGLADESH}

\author{
MR Amin (1), SMH Mamun (2), M Rahman (3), A Ghose (4), R Rahman (5), MA Faiz (6). \\ (1) Hathazari Health Complex, Chittagong, Bangladesh; (2) Chittagong Medical College Hospital, Bangladesh; (3) Shahabuddin Medical \\ College, Dhaka, Bangladesh; (4) General Hospital, Chittagong, Bangladesh; (5) Begum Khaleda Zia Medical College, Dhaka, Bangladesh; \\ (6) Dhaka Medical College, Bangladesh
}

Objective: Snake bite is a potentially life threatening emergency situation that physicians encounter in rural areas of tropical countries in South-East Asia including Bangladesh. Different variable neurological manifestations of venomous snake bite are seen 
around the world. Among the venomous snakes in Bangladesh, neurotoxic snakes like Cobra and Krait are the commonest. In this study neurotoxic manifestations of venomous snakes were clinically observed.

Methods: In this series a total 35 snakebite patients with neurological features from May 1999 to June 2001 were included and preexisting neurological cases were excluded.

Results: Among the 537 total snake bite cases, the neurotoxicity due to snake bite was $10 \%$ with 51 cobra bites and 12 krait bites. The victims' ages were in the range of 3.5 years to 85 years with $70 \%$ of cases under 30 years of old. There is slight male preponderence with almost same number of bites at home and outside. The common clinical neurotoxic features are ptosis (100\%), external ophthalmoplegia, dysphagia, dysphonia and broken neck sign. The chest movements were reduced in $20 \%$ of cases. All 35 cases $(100 \%)$ were treated with Haffkine polyvalent anti-snake venom with $8.6 \%$ of cases needing a 2nd dose. All 35 cases with neurotoxic features were also treated with anticholinesterases (100\%) and among them $14.2 \%$ needed ventilatory support. Antivenom reaction was very common in the pyrogenic reaction (80.64\%) and anaphylactic reaction (64.51\%). The outcome of snake bite was excellent with $97 \%$ recovery with one residual neurological deficit and no fatality.

Conclusion: The neurotoxic snake bite has definite characteristic neurological signs and symptoms which could lead to fatality with respiratory paralysis.

\title{
PP 100. EFFECT OF SEASONAL VARIATION (RAIN) UPON MORTALITY FROM YELLOW OLEANDER SEEDS POISONING
}

\author{
C Palangasinghe, L Senarathna, AH Dawson. \\ South Asian Clinical Toxicology Research Collaboration, University of Peradeniya, Sri Lanka
}

Objectives: Deliberate self poisoning with yellow oleander seeds (Thevetia peruviana) is common in rural Sri Lankan districts. The cardiac glycosides in oleander seeds cause cardiac arrhythmias leading to death from ventricular arrhythmia. The toxicity following ingestion depends upon the level of glycoside in the seed and extents of absorption. The maturity may depend on the environmental factors such as rain. Over few years, as a part of large cohort study, a varying cardiac toxicity was observed over the months. This data analysis was designed to identify variation of cardiac toxicity and potential relationships with rainfalls.

Methodology: Data was collected from oleander patients from mid 2002 together with their outcome and with details of special treatments like cardiac pacing. Mortality and percentage received cardiac pacing were calculated on a monthly basis. There were data from 54 months. Depending on the data of the monthly average rainfall from meteorology department the patients were categorized according to the rainfall of the month they admit. The raining pattern of consecutive months was compared with changing mortality/ cardiac toxicity to identify possible relationships. Multiple logistic regression methods were used to adjust this relationship with other factors such as number of seeds, age and gender.

Results: There were 3546 yellow oleander admissions to two study hospitals during 54 months. There were 147 deaths (4.7\%) and 80 (2.26) patients underwent temporary cardiac pacing after transferring to tertiary hospitals. The rainfall varied from 0 to 750 mm with median of $67 \mathrm{~mm}$ (IQR 26-167) during these 54 months. The highest number of admission 1432 (40.38\%) and highest monthly average admissions (57) were observed when rainfall is below $50 \mathrm{~mm}$. The highest number of deaths (117/147) had occurred when the rainfall was below $150 \mathrm{~mm}$. The OR of dying is 1.5 (95\% CI 1.05, 2.14) during low rainfall (below 100mm) but when adjusted with other confounding factors such as age, gender and number of seeds ingested by using multiple logistic regression method, the OR became 1.41 (95\% CI 0.97, 2.05).The OR for temporary cardiac pacing shows non-significant relationship when adjusted with above factors (OR 1.1 [95\% CI 0.70, 1.77).

Conclusion: Rainfall influenced the number of oleander poisoning admissions but was not clearly related to mortality or need for pacing. Regional and referral hospitals should be prepared for higher number of admissions in drier months.

\section{PP 102. VITAL ISSUES FACED WHILE TREATING KRAIT (BUNGARUS CAERULEUS) BITE VICTIMS BY A TERTIARY HOSPITAL IN SOUTH INDIA-CONFOUNDING BRAIN STEM DYSFUNCTION SIGNS AND IS THERE AN UPPER LIMIT TO ASV DOSAGE HERE?}

\author{
R Thirumavalavan. \\ Department of Critical Care and Clinical Toxicology, SRM Medical College Hospital and Research Center, Kattankulathur, Kancheepuram Dt, \\ Tamil nadu State, India
}

Background: SRM Medical College Hospital \& Research Center is situated in South India and surrounded by 112 villages. Snake envenomation is common and the ICU often has to give life support to these victims. 


\begin{abstract}
Aim: Clinical assessment of persistent brain death is usually taken as sign of poor survivability and decision is taken to discontinue life support. But if this is due to Krait envenomation, it could be totally reversible. Krait (Bungarus caeruleus) is a nocturnal snake prevalent in India. Bite typically occurs in night in a village while victim is lying on the floor with door open, is often painless with no cellulitis, progressing to neuroparalysis, all of which makes this the most lethal snake in India. We share our experience in treating a series of Krait envenomations, where despite absent brain stem reflexes, patients continued to receive Criticare including ASV and ventilatory support resulting in return of brain stem signs and all patients discharged home in a neurologically viable condition. Also we followed the latest WHO protocol and did not exceed $8+8$ vials while treating these victims despite continued ventilatory dependence taking into account the early presynaptic depletion of acetylcholine.

Methods: Patients presenting to ER who had a fresh neurological deficit who were apparently normal previously were shifted to ICU. Routine blood tests done. 3 patients were brought "brain dead". All had weakness with areflexia and hypotonia. ASV 8 Vials given immediately followed by another dose an hr later. Respiration supported by mechanical ventilation.

Results: All Krait victims had only neurotoxicity and normal hematological values. Life support not abandoned despite persistent coma with GCS 3/15 and absent brain stem reflexes for even 2 to 4 days. All recovered over 2 to 4 days on ventilation, and after an average 8 days stay were discharged neurologically viable with normal motor power.

Conclusions: Krait is an Elapid and causes only neurotoxicity which may develop into coma with absent brain stem reflexes which is totally reversible with ASV and mechanical ventilation, and this should be considered in differential diagnosis. Life support is mandatory anticipating return of neurological status. Due to presynaptic inhibition in Krait it is redundant to give ASV beyond 2 doses of 8 vials each totaling 16 vials. We have to merely continue life support and Improvement takes place in due course.
\end{abstract}

\title{
PP 103. PUFFER FISH POISONING FROM ILLICIT FISH TRADING IN BANGKOK, THAILAND
}

\author{
S Linlawan (1), S Suteparuk (2). \\ (1) Department of Medicine, King Chulalongkorn Memorial Hospital; (2) Department of Medicine, Faculty of Medicine, Chulalongkorn \\ University, Bangkok, Thailand
}

Background: Many species of marine puffer fish contained tetrodotoxin (TTX). Ingestion of these fish leads to muscle paralysis and even death in severe cases. Recently there have been about 75 tons of puffer fish meat illegally streamed into fish markets of Thailand everyday. They were sold as sliced fish in the names of other fish. Many consumers take puffer fish without recognition.

Objective: To report the recent incidents of puffer fish poisoning in Bangkok.

Case series: From June to August 2007, we found eight patients with clinical compatible with tetrodotoxin intoxication in King Chulalongkorn Memorial Hospital. Three, one and four cases were distributed among three incidents. All cases had the history of eating and buying food containing sliced fish from food vendors. Later the authorities identified the fish in the first and third incidents to be puffer fish. The cases were 2 males and 6 females, aged from 20 to 55. One patient, a 50-year-old female, had ventilatory failure and needed ventilatory support. Another patient, a 21-year-old female, complained of difficulty of walking and had grade III-IV/V generalized muscle weakness. The others had minor symptoms such as numbness, dizziness, nausea and vomiting. Five cases needed hospitalization. All patients recovered uneventfully within 24-48 hours of supportive treatment.

Conclusion: Eight patients with puffer fish poisoning were reported in our hospital in a span of less than three months. They had good clinical outcome though one case could be fatal if prompt treatment had not been given. Due to the large amount of illicit puffer fish trading, more cases are expected. Laws enforcement and public acknowledgement are mandatory.

\section{PP 073. ACUTE VITAMIN A POISONING FOLLOWING FISH LIVER INGESTION}

CF Huang (1), CC Yang (1,2), JF Deng (1).

(1) Division of Clinical Toxicology, Taipei Veterans General Hospital, Taipei, Taiwan; (2) Department of Environmental and Occupational Medicine, National Yang-Ming University, Taipei, Taiwan

Background: Toxic levels of vitamin A may accumulate in the livers of certain fish species, such as sharks, snappers, tuna and sea basses. Ingestion of large amounts of fish liver therefore can cause vitamin A poisoning, an illness characterized by severe headache, fever and desquamation. Although fish liver ingestion could cause vitamin A poisoning, human poisoning cases are rarely reported.

Methods: We retrospectively reviewed the records of patients with suspected fish liver poisoning reported to the Taiwan Poison Center (PCC-Taiwan) from July 1986 through April 2007. Patient's age, gender, reason of exposure, time of exposure, ingested fish species, time elapsed after exposure, clinical manifestations, treatments, and outcomes were collected and analyzed. 
Results: A total of 6 incidents involving 19 patients were identified. Males outnumbered females (68\% vs. 32\%) in vitamin A poisoning related to fish liver ingestion. The culprit fish species were tuna in 8 patients ( 1 incident), sea bass in 1 patient, snapper in 3 patients ( 1 incident), and unknown fish species in 7 patients ( 3 incidents). Following the consumption of fish liver, all patients developed gastrointestinal and neurological effects, such as nausea, dizziness, headache, and weakness, within 4 to 12 hours. Some patients also manifested fever, facial flush, hypertension, and conjunctival congestion. Diffuse desquamation and/or hair loss, which might last up to 8 weeks, developed after the resolution of the aforementioned manifestations within 1 to 4 days. All of the patients recovered well with supportive therapy alone.

Conclusion: Ingestion of fish livers that are rich in vitamin A can result in moderate to severe poisonings. Although it is not possible to completely exclude the coexistence of ciguatera poisoning in some of the patients, the major effects of fish liver poisoning in this study resembled those of acute hypervitaminosis A from overdosage of dietary vitamin A supplements. Prompt diagnosis of acute vitamin A poisoning can be difficult in the absence of diffuse desquamation because the clinical manifestations are indistinguishable from ciguatera poisoning and routine bioassay is unavailable. Management of vitamin A poisoning related to fish liver ingestion is primarily symptomatic and supportive, and the outcome is generally favorable.

\title{
PP 005. TETRODOTOXIN POISONING IN BANGLADESH: A CASE STUDY
}

\author{
A Ghose (1), H Ahmed (2), A Basher (2), MR Amin (3), AA Sayeed (4), MA Faiz (2). \\ (1) Chittagong General Hospital, Chittagong, Bangladesh; (2) Dhaka Medical College Hospital, Dhaka, Bangladesh; (3) Hathazari Health Complex, \\ Chittagong, Bangladesh; (4) Chittagong Medical College Hospital, Chittagong, Bangladesh; (5) Dhaka Medical College Hospital, Dhaka, Bangladesh
}

Objective: Puffer fish consumption and as a consequence tetrodotoxin poisoning out of it is becoming an increasingly common incidence in Bangladesh. Due to availability and affordability in rural areas puffer fish ingestion is becoming an important cause of poisoning mainly in the coastal regions of the country. In this communication we report an event of tetrodotoxin poisoning in ten people in Bangladesh.

Case report: Nine male and one female patients were admitted to Dhaka Medical College Hospital with a history of ingestion of puffer fish. Three of them were from same family; others are from same locality but not related. Presenting features were tingling sensation around mouth and lower limbs in 7 patients, heaviness of tongue in 5 , weakness in walking in 6 , vomiting in 9 , blurring of vision in 2 , double vision in 1, inability to talk, slurred speech and generalized weakness in 2 , respiratory difficulty in 2 patients. Sensory symptoms were reported to appear earlier. Physical examination revealed reduced touch sensation in both upper and lower limbs and in face in 3 patients, dilated pupils slowly reacting to light in 5 patients. One patient presented with respiratory failure with muscle power $1 / 5$, absent deep jerks and reflexes and died within 20 minutes of admission. Five other patients were treated with subcutaneous neostigmine. Remaining patients received symptomatic management. All nine patients improved and were discharged after one day. Patients were not available for follow up.

Conclusion: Health personnel should be aware and have sufficient knowledge regarding the manifestations, complications and management of puffer fish poisoning. Common people should be made aware of the potential risk of eating puffer fish, proper preparation process, about the warning symptoms and signs of puffer fish poisoning, and when to seek medical help.

\section{OP 028. A RANDOMISED CONTROLLED TRIAL OF INTRAMUSCULAR VERSUS INTRAVENOUS ANTIVENOM FOR REDBACK SPIDER BITE-THE RAVE STUDY}

\author{
GK Isbister (1,2,3), SGA Brown (4,5), M Miller (6), A Tankel (7), E MacDonald (3), R Ellis (8), Y Nagree (9), G Wilkes \\ (10), $R$ James (2), B Stokes (3), A Holdgate (11), A Short (11). \\ (1) Menzies School of Health Research, Charles Darwin University, Darwin; (2) Calvary Mater Newcastle; (3) Faculty of Health, University of \\ Newcastle, NSW; (4) Emergency Department, Fremantle Hospital, Perth; (5) Emergency Medicine Research Unit, Discipline of Emergency \\ Medicine, University of Western Australia; (6) Emergency Department, John Hunter Hospital, Newcastle; (7) Emergency Department, Coffs \\ Harbour Hospital; (8) Emergency Department, Rockingham-Kwinana District Hospital, Rockingham; (9) Emergency Department, Armadale- \\ Kelmscott Memorial Hospital, Armadale; (10) Emergency Department, Bunbury Regional Hospital, Bunbury; (11) Emergency Medicine Research \\ Unit, Liverpool Hospital and University of New South Wales
}

Background: Widow spider-bite causes latrodectism and is associated with significant morbidity worldwide. We aimed to compare the effectiveness of intravenous (IV) versus intramuscular (IM) widow spider antivenom.

Methods: Patients with latrodectism were given either IV or IM antivenom according to a randomized double-dummy, doubleblind protocol. The first antivenom treatment was followed by another identical treatment after two hours if required. The primary 
outcome was a clinically significant reduction in pain two hours after the last treatment. A fully Bayesian analysis was used to estimate the probability of the desired treatment effect, predetermined as an absolute difference of $20 \%$.

Findings: We randomly allocated 126 patients to receive antivenom IV(64) and IM (62). After antivenom treatment pain improved in 40/64 (62\%) in the IV group versus 33/62 (53\%) in the IM group (+9\%; 95\% Credible Interval[CrI]: $-8 \%$ to $+26 \%$ ). The probability of a difference greater than zero (IV superior) was $85 \%$ but the probability of a difference greater than $20 \%$ was only $10 \%$. In 55 patients with systemic effects, pain improved in 58\% after IV antivenom versus 65\% after IM antivenom (-8\%; 95\%CrI: $-32 \%$ to $+17 \%$ ). Twenty four hours after antivenom pain had improved in $84 \%$ in the IV group versus $71 \%$ in the IM group (+13\%; $95 \%$ CrI: $-2 \%$ to $+27 \%)$. A meta-analysis including data from a previous trial found no difference in the primary outcome between IV and IM administration.

Conclusion: The difference between IV and IM routes of administration of widow spider antivenom is, at best, small and does not justify routinely choosing one route over the other.

\title{
OP 029. THE IMPACT OF SNAKEBITE ON HOUSEHOLD ECONOMY IN BANGLADESH
}

\author{
SMK Hasan (1), A Ali (2), MA Jalil (3), MA Faiz (1), Dhaka University, Dhaka Bangladesh. \\ (1) Department of Medicine, Dhaka Medical College; (2) Institute of Health Economics; (3) Department of Statistics
}

Objectives: The amount of wealth loss due to mortality and morbidity from snakebite can hamper household economy. The present study aims to assess different types of costs for treatment of snakebite patient, quantify household economic impact and understand the coping mechanism to cover the costs of snakebite patients in Bangladesh.

Methods: A total of 83 snakebite patients admitted from June 2006 to October 2006 for treatment in four tertiary level hospitals in Bangladesh in Dhaka, Chittagong, Khulna, and Rajshahi. The patients were interviewed using structured questionnaire documenting history, clinical features, treatment seeking behavior, health care related expenditures and the way in which the expenditures were covered. Cost related to snakebite included direct cost (service provider fees, drugs, diagnostic cost and others), indirect costs (transport, attendant cost, food cost etc.), and opportunity costs (wages loss).

Results: Among the patients $71.1 \%$ were male, $54.2 \%$ of the snakebites were nonvenomous and $45.8 \%$ were venomous. Occupation of the patients were agricultural labor (18.1\%), housewives (16.5\%), business (15.7\%) and service (14.5\%). Snake bite occurred during walking on the way (22.9\%) and during sleeping (16.9\%). 68.7\% of the patients have seen the snake and among them $22.9 \%$ could mention the name of the snake. Majority (65.1\%) of the patients went to visit a traditional healer (Ohzas). A significant number (92.8\%) of the patients used tight tourniquet after snakebite. Bite marks were visible in $60.2 \%$ of the patients ( $\mathrm{n}=50$ ). Among the patients $42.2 \%$ were given polyvalent antivenom. Total expenditure related to snakebite varies from BD Taka 442 (US I \$ $=$ Taka 67) to 153700 (US\$) with a mean of BD Taka 8305 and mean income loss was BD Taka 6244. Expenditure for venomous snakebite (BD Taka 15479) is about 7 times higher than nonvenomous snakebite (BDTk.2248). Among the households with snakebite $61.4 \%$ lend money to meet up the cost of the treatment. A very few of them mortgaged land and business. 3.6\% $(\mathrm{n}=3)$ sold ornaments and $4.8 \%$ $(\mathrm{n}=4)$ sold livestock to compensate the treatment cost.

Conclusion: The treatment of snakebite patients causes a major economic burden in affected families especially in venomous snakebite cases. This may impoverish the family and affect the other household expenditures for education, food and nutrition. Appropriate measures should be launched for prevention and treatment of snakebite in vulnerable areas to prevent gained economic loss and valuable life.

\section{OP 030. THE PRODUCTION OF BUNGARUS CANDIDUS ANTIVENOM FROM HORSES IMMUNIZED WITH VENOM AND ITS APPLICATION FOR TREATMENT OF SNAKE BITES IN VIETNAM}

\section{K Xuan Trinh, D Pham.}

The Venom Research and Antivenom Production Unit (VRU), The National Poison Control Center (PCC) of Vietnam, Bach Mai Hospital, Hanoi, Vietnam

Introduction: Administration of antivenom (AV) has been for many years the most widely and effectively accepted form of treatment of snakebites (WHO, No 463,1971). Unfortunately, Bungarus candidus (BC) is the most venomous snake in the land. Their mortality rate is $80 \%$ in the severe envenoming patients. However, there is not specific $B C$ AV available in the world. It should be made $B C$ AV to save the life of $B C$ snakebite patients in Vietnam.

Objective: To research and make $B C A V$ for treatment of $B C$ snakebite patients in Vietnam. 
Methods: $B C$ venom was detoxified by glutaraldehyde to make $B C$ antigen. The horses were immunized by this antigen combined with complete and incomplete Freund adjuvant. The specific antibody response of the horses was detected by Ouchterlony and ELISA. Plasma from them was extracted every month. Method used for fractionation was immunoglobulin digestion by pepsin to produce $\mathrm{F}\left(\mathrm{ab}^{\prime}\right)_{2}$ fragments AV. Patients were bitten by $B C$ were treated by this $B C \mathrm{AV}$.

Results: Three immunized horses were responsed of maximal antibody at the 7 th month after immunization. The $B C \mathrm{AV}$ was produced by VRU and passed quality control of the National Institute for Control of Medico-Biological Products, MOH, Vietnam. $90 \mathrm{BC}$ patients with severe envenoming required artificial ventilation were treated by $B C \mathrm{AV}$. 24 hours after the $B C$ AV treatment, all of them were recovered completely with 5\% side effects, compared to the corresponding group, but did not get the $B C$ AV treatment: Mortality was $80 \%$ and the times of artificial ventilation were one month or more.

Conclusion: $B C \mathrm{AV}$ was made and applied successfully for the treatment of $B C$ snakebite patients in Vietnam. It is the first $B C$ AV in the world.

\title{
OP 031. CURRENT USE OF AUSTRALIAN SNAKE ANTIVENOMS AND FREQUENCY OF ADVERSE REACTIONS
}

\author{
GK Isbister (1,2), SGA Brown (3,4), E MacDonald (3), I White (5), BJ Currie (1) the ASP Investigators. \\ (1) Menzies School of Health Research, Charles Darwin University; (2) Calvary Mater Hospital, Newcastle; (3) Emergency Department, \\ Fremantle Hospital; (4) Emergency Medicine Research Unit, Discipline of Emergency Medicine, University of Western Australia; (5) Toxicology \\ Department, Women's and Children's Hospital, North Adelaide and Adelaide University, South Australia
}

Objective: To investigate current use of Australian snake antivenoms and the frequency and severity of immediate-type hypersensitivity reactions.

Methods: This was a nested prospective cohort study of patients receiving snake antivenom treated in Australian hospitals from January 2002 to August 2007 as part of the Australian Snakebite Project-ASP. The outcomes were use of antivenom, frequency and severity of hypersensitivity reactions to antivenom and treatment of these reactions.

Results: One hundred and sixty nine patients received snake antivenom. The commonest reason for antivenom administration was venom induced consumption coagulopathy in 123 cases (73\%), followed by non-specific systemic effects (14\%), neurotoxicity(5\%) and myotoxicity (4\%). In eight patients(5\%) antivenom was given in non-envenomed patients. The commonest antivenoms used were brown snake (46\%), tiger snake (31\%) and polyvalent (12\%). The median dose was 4 vials (IQR: $2-5$ vials) and 20 patients received two different types of antivenom. Hypersensitivity reactions occurred in 43 cases (25\%); 19 satisfied our definition of anaphylaxis, of which 10 were moderate and 9 were severe including 8 that were hypotensive (systolic BP $<90 \mathrm{mmHg}$ ). The remaining 24 reactions were mild (skin only). Adrenaline was used for treatment in 25 cases. The reaction rate was higher for tiger snake (42\%) and polyvalent (45\%) antivenoms than for brown snake antivenom (10\%). Hypersensitivity reactions occurred in 9 of 32(31\%) patients receiving any form of premedication versus 16 of 62 (26\%) not receiving premedication.

Conclusions: Antivenom was used appropriately in most cases with coagulopathy being the commonest indication. Hypersensitivity reactions were common, however, more than half were only mild. The discretional use of premedication was not associated with any reduction in reaction risk.

\section{OP 046. A DOSE ESCALATION STUDY TO DETERMINE THE SAFETY AND EFFICACY OF FRUCTOSE 1,6-DIPHOSPHATE (FDP) IN TREATING YELLOW OLEANDER-INDUCED CARDIAC TOXICITY}

\author{
M Fahim (1), M Perera (1), I Gawarammana (1), S Jayamanne (2), H Karunathilake (3), C Abayasinghe (3), \\ N Buckley (1, 4), A Dawson (1). \\ (1) SACTRC, Faculty of Medicine, University of Peradeniya, Sri Lanka; (2) General Hospital, Polonnaruwa, Sri Lanka; (3) General Hospital, \\ Chilaw, Sri Lanka; (4) Department of Clinical Pharmacology and Toxicology, Canberra Clinical School, ACT, Australia
}

Objective: The principle objective is to find the lowest dose of FDP that has a beneficial effect on yellow oleander-induced cardiac toxicity in human.

Background: Cardiac glycoside toxicity is one of the most common causes of drug-induced toxicity and is the most common type of plant poisoning in Sri Lanka and some other South Asian countries. The only current antidote (Digoxin Fab antibody fragments-DigiFab-KaneruTab) is effective but extremely expensive. Fructose-1,6-diphosphate (FDP) has been shown to treat cardiac 
toxicity in animal studies of oleander poisoning and has been used intravenously in a large number of human studies. It has the advantages of being cheap and low human toxicity.

Method: We have conducted a multi-centre phase II dose escalation study in the General hospitals of Polonnaruwa and Chilaw for the period of 16 months (April 2006 to July 2007). Patients presenting with significant cardiotoxicity after ingestions of oleander seeds were recruited to this study. Informed consent was obtained from all patients. Patients were randomly allocated to four dose levels doubling at successive dose levels $(30,60,125$, and $250 \mathrm{mg} / \mathrm{kg})$. In each dose level two patients received placebo and six patients received active treatment. Blood samples, serial ECG and continuous holter readings were obtained.

Results: We have recruited 32 patients; 20 were female (62.5\%). Median age and number of oleander seeds ingested were 25 years (IQR 18-34) and 3 seeds (IQR 2-5) respectively. Median time from ingestion to starting of the FDP/placebo was 12 hours (IQR 6-19.5). Holter reading was obtained (mean duration 121. 25 minutes) from all the patients and sent to our collaborators in Australia for interpretation. There were two deaths $(6.25 \%, 95 \%$ CI 1.1-19.2), one in each study group. Both these patients died due to cardiac arrest. FDP was tolerated well in our patients. None of them develop major adverse effect or allergic reactions to FDP. Two patients developed transient hypotension on completion of bolus infusion of FDP which responded to fluids and ionotropes.

Conclusion: This study does confirm that FDP even at a higher dose of $250 \mathrm{mg} / \mathrm{kg}$ in severe cardiac toxic patients is safe. FDP may be effective in reducing deaths on oleander toxicities. However further studies are needed to confirm our preliminary findings.

\title{
IS 023. MASS CASUALTY OF BOTULISM: CLINICAL AND NEUROLOGICAL FINDINGS
}

\author{
$R$ Witoonpanich. \\ Division of Neurology, Department of Medicine, Faculty of Medicine, Ramathibodi Hospital, Mahidol University, Bangkok, Thailand
}

Botulism is a rare presynaptic neuromuscular junction disorder caused by potent toxins produced by anaerobic, spore-forming, grampositive bacteria Clostridium botulinum. The clinical syndrome of botulism is dominated by neurological symptoms and signs resulting from toxin-induced blockade of acetylcholine release at the cholinergic neuromuscular junction, sympathetic and parasympathetic ganglia and parasympathetic post-ganglionic sites. C. botulinum is ubiquitous being present mostly in soil throughout the world. However, growth and elaboration of toxin occur only under particular conditions that include an anaerobic, low-salt and low-acid environment.

Food-borne botulism is caused by ingestion of foods contaminated with botulinum toxin and outbreaks have been reported from various countries. Outbreaks may be small (involving a few persons) or large but sporadic cases do occur. These have been associated with home-preserved or home-canned food, mostly vegetables or traditionally prepared food, improperly stored commercial or noncommercial food and fermented or dried fish and sea mammal products. A large outbreak occurred during mid-March 2006 in Nan, Thailand affecting about 200 people following ingestion of home-canned bamboo shoots at a religious rite in a village. This has been the largest single outbreak of food-borne botulism in the world to date. One hundred and fifteen patients were admitted to the provincial hospital. Most of the cases first presented with gastrointestinal (GI) symptoms within several hours to a few days after ingestion of the food followed by neurological symptoms. The incubation periods estimated as median time from ingestion of food to GI symptoms was 24.5 hours, median time to neurological symptoms was 48 hours and median time to endotracheal intubation was 72 hours. The GI symptoms included nausea, vomiting, abdominal pain, flatulence, diarrhea and constipation. The most striking and early neurological symptom and sign was difficulty in swallowing. Other common signs were ptosis and ophthalmoplegia. The latter involved all extraocular muscles in some cases and only one or two muscles in others with lateral rectus being the most common muscle to be affected. Other signs included predominantly proximal weakness of the extremities and pupillary abnormality. Pupils were dilated and fixed or sluggishly reactive to light in severe cases. Forty-two cases had respiratory failure requiring mechanical ventilation which was preceded by history of difficulty in breathing in about one half of the patients.

All patients had antitoxin injection. There was no mortality in this outbreak and all patients steadily recovered. It was noted that ptosis and ophthalmoplegia resolved rapidly within 1-2 weeks except in very severe cases. Difficulty in swallowing, weakness of extremities and respiratory impairment took longer to subside. Even if the muscle power was full on testing, some patients still complained of fatigue for some time. The overall clinical course expressed as median time on ventilator and in hospital was 14 days for both. 